\title{
THE DISCOVERY OF THE CHEMICAL COMPOSITION - KINEMATICS CONNECTION IN THE 1950'S
}

\author{
NANCY GRACE ROMAN \\ Hughes STX (NASA Astronomical Data Center) \\ Greenbelt, Maryland, U.S.A. \\ (roman@nssdc.gsfc.nasa.gov)
}

\begin{abstract}
.
This paper reviews the recognition that kinematic characteristics of stars are related to their spectroscopic appearance on low and intermediate dispersion spectra. These differences result from the differences in the abundances of metals as determined from high dispersion spectra. Presumably, these abundance differences reflect differences in age. The highvelocity carbon stars share the molecular peculiarities of the high-velocity oxygen giants but, because of the greater strength of the bands of carboncontaining molecules, exhibit them to a greater degree.
\end{abstract}

\section{Introduction}

In his classic paper on the high-velocity stars, Oort (1926) noted that the luminosity curve for the high-velocity stars was appreciably steeper than that for the low-velocity stars. He also noted that very few early-type stars were included among the high-velocity stars. All of the globular clusters have high velocities as do $75 \%$ of the RR Lyrae stars, according to Oort. Miczaika (1940) showed that the HR diagram for the high-velocity stars differs significantly from that of the majority of the stars near the Sun or of open clusters, noting that stars earlier than about F5 are scarce and that the brightest stars are red giants about photographic magnitude $=-1$ with a strong population just below that.

When Baade (1944) discovered that the brightest stars in M32, NGC 205, and the nucleus of M31 are red giants with an absolute magnitude near -2 with no bright blue stars among them, it was natural to associate 
them with the globular clusters and with high-velocity stars. The globular clusters also contain RR Lyrae stars that in the field have high velocities, and both globular clusters and RR Lyrae stars are found at a large distance from the plane in the Milky Way and in other galaxies. Thus, in spite of substantial evidence to the contrary by 1955 , the high-velocity stars were all normally assigned to Population II.

\section{F-type Subdwarfs}

The first reference to a relation between spectral types and peculiarities and velocity appears to be in a 1914 paper by Adams and Kohlschütter (1914). They state:

"An examination of the spectral types of the stars with great velocities shows that nearly all classes are represented among them. An interesting fact, however, is that the two stars with the largest velocities of all are of types F3 and F7 and the two stars succeeding these are of the A type. The spectra of these last two stars Lal. 5761 [HD19445] and Lal. 28607 [HD140283] are both peculiar in that the magnesium line $\lambda 4481$ is either absent or extremely faint, while in other respects the spectra are of the normal A2 or A3 type. The remarkably high and nearly equal velocities for two stars of nearly $i$ dentical but peculiar spectra form a singular coincidence. ... The existence of high radial velocities among stars having what is generally considered an early type of spectrum is shown by these results although there can be no doubt that such cases are rare."

Four additional stars of this type were discovered in the course of a radial velocity program (Adams et al., 1935).

Chamberlain and Aller (1951) published a detailed comparison of the spectra of two of the subdwarfs with that of the normal star, 95 Leonis. They found that both subdwarfs could be represented by an F-type model atmosphere with a temperature near $6300 \mathrm{~K}$ but abnormally small amounts of calcium and iron compared to hydrogen. They suggested that these highvelocity subdwarfs might be related to the bright weak-line stars discussed by Roman (1950). Although the results were somewhat uncertain, M. and B. Schwarzschild (1950) thought that the $\mathrm{H} / \mathrm{Fe}$ ratio might be somewhat higher in these high-velocity stars than in low-velocity stars. They noticed that $\mathrm{CH}$ was definitely stronger and suggested that $\mathrm{C}$ was probably about 2.5 times more abundant in the high-velocity subdwarfs than for most high-velocity stars. Chamberlain and Aller had stated that measures of the Balmer discontinuity were desirable. These were provided in by Berger (Berger et al., 1951). He stated:

"The discontinuity of the Balmer $\mathrm{D}$ is clearly smaller for the subdwarfs than for normal stars of the same type. ... In spite of the uncertain character of 
these measurements, ... they permit the enunciation of the conclusion that the color temperature of the continuous spectrum (blue-violet) of a subdwarf of type $F$ is less than that of a normal star of the same type."

One of the stars was illustrated in the Yerkes spectral atlas (Morgan et al., 1943) where its resemblance to the RR Lyrae stars was noted.

In the course of a study of high-velocity stars (Roman, 1954), $17 \mathrm{~F}$ type subdwarfs were defined as having $\mathrm{B}-\mathrm{V}$ between 0.00 and +0.80 , sharp hydrogen lines of about the same strength as those in middle F-type stars, the $K$-line approximately equal in strength to $\mathrm{Ca} \mathrm{H}+\mathrm{He}$, and the $\mathrm{G}$-band, $\mathrm{CaI} \lambda 4226$ and the strongest of the metallic lines absent or present weakly. Although the B-V colors of these stars agree well with their spectral types, the U-B colors are systematically too negative by nearly 0.2 magnitudes. Reiz (1954) showed that the position of these stars in the HR diagram could be explained by a stellar model with negligible heavy elements. The comparatively bright ultraviolet is, presumably, also due to the scarcity of the metallic lines. About the same time, Schwarzschild, Searle, and Howard (1955) came to the same conclusions from an analysis of higher resolution spectra.

The most interesting characteristic of these stars is the nature of their galactic orbits. The space velocities are much higher (relative to the Sun) than those of most high-velocity stars, indicating almost no galactic rotation. The eccentricities are very high, with most of the stars penetrating the galactic bulge. Their velocities perpendicular to the the galactic plane are similar to those of the globular clusters and the RR Lyrae stars. Hence, these stars are clearly classical Population II objects. It would be surprising if they are not present in globular clusters.

\section{Late-type Giants}

Lindblad (1922) obtained spectra of the three brightest stars in M13. He estimated the types to be near $\mathrm{K} 0$ and the absolute magnitudes near -3 . However, he noted that the absorption in the region of the cyanogen band is much smaller than in the late-type stars in M11. Morgan and Keenan in the $M K K$ Atlas (1943) also noted that the $C N$ absorption is abnormally weak on low dispersion spectra of three or four high-velocity stars. Fourteen low dispersion spectra of G- and K-type giants in M13 were studied by Popper (1947). He concluded that the CN absorption is indeed weak in these stars, both blueward of $\lambda 4215$ and near $\lambda 3883$. The strength of the $C N$ in these features is comparable to that in class IV stars although the atomic lines indicate a luminosity class near $\mathrm{Ib}$, more consistent with the absolute magnitudes of the stars.

Keenan, Morgan, and Münch described the spectra of high-velocity 
giants in detail (1948) as follows:

"In types $G$ and $K$ the bands of $\mathrm{CN}$ are definitely weaker in high-velocity stars than in ordinary stars of the same luminosity. The stronger metallic lines appear nearly normal in the high-velocity stars. The Balmer lines of $\mathrm{H}$ and the $G$-band ( $\lambda$ 4300) of $\mathrm{CH}$ are at least as strong as in ordinary stars and may be slightly strengthened in high-velocity stars.

Among the carbon stars (Type R) the high-velocity ones are marked by such tremendous strengthening of the G-band that they have been called $\mathrm{CH}$ stars. The CN-bands appear on the average to be somewhat weaker than in ordinary carbon stars. The Balmer lines and $\lambda 4554$ of $\mathrm{Ba}+$ are slightly strengthened."

In both of these groups the peculiarities shown by the high-velocity stars are more pronounced in molecular features $(\mathrm{CH}, \mathrm{CN})$ than in atomic lines. The elements most affected are $H, C, N$, and possibly $O$ (because of the great influence of $C O$ abundances on the equilibrium concentration of $C N$ ).

There were several papers on the spectra of high-velocity stars in 1950 . Iwanoska (1950) compared 22 high-velocity and 15 standard stars spectrophotometrically. She concluded:

"General weakening of lines is observed in most high-velocity stars of spectral class $G$ and $K$ " and

"Little or no weakening is observed for the lines of $\mathrm{Sc}+, \mathrm{V}, \mathrm{Ti}, \mathrm{Cr}$, ionized rare earths and $\mathrm{CH}-$ band."

Miczaika (1950) confirmed the weakening of the $C N$ and possible strengthening of the $\mathrm{CH}$; he found no difference in the equivalent widths of the iron lines. Schwarzschild, Spitzer, and Wildt (1951) showed that the behavior of the bands could be explained by a decrease in the abundance of the metals and a smaller decrease in the abundance of oxygen. Thus, although the effects in the atomic spectra are less striking in the late giants than in the F-type stars, the cause is the same.

Baum (1952) showed that spectra of the bright giants in M3 and M92 show the same metallic-line weakness as do the high-velocity stars, with the weakness particularly great in M92, but the cluster stars show the weakening of the metallic lines to a much greater degree (Keenan and Keller, 1953). The latter authors pointed out, however, that none of the high-velocity stars they studied are very similar to the stars observed by Baum. Keenan and Keller noted the same spectral peculiarities in the high-velocity stars that were described previously. They suggested that the ratio of $\lambda 4172 / \lambda 4216$ provides a useful spectroscopic criterion for recognizing high-velocity stars and demonstrated a clear correlation of this ratio with velocity. $\lambda 4172$ may be strengthened by the weakening of the partially overlapping $C N ; \lambda 4216$ is a blend of $S r$ II and $C N$. Gratton (1954) pointed out that the high and low-velocity giants are not two homogeneous groups of stars; as far as chem- 
ical composition is concerned, the fluctuations within each group are of the same order as the differences between them.

\section{Old Population I Stars}

In 1950 Vyssotsky reported in the IAU Transactions that in some G-type stars both the G-Band and $H \gamma$ are strengthened simultaneously. Moreover, the strengthening seemed to be correlated statistically with larger than average proper motions but did not appear to be a luminosity effect.

While classifying the northern F- and early G-type stars brighter than 5.5, Roman (1950) noticed differences in the intensities of the metallic lines for stars of the same spectral type. In classifying the later type stars she (1952) discovered that it was possible to detect spectroscopic differences in these also, which are unrelated to temperature or luminosity. The large majority of these later stars can be differentiated by the strength of $\lambda 4226$ of $\mathrm{CaI}$ relative to the strength of $\lambda 4045$ of $\mathrm{FeI}$. The G-band also appears stronger in the stars with strong $\lambda 4226$. This is the same effect as noticed by Vyssotsky of which she was unaware until recently. It is clear from the velocity distribution that these groups correspond to the strong- and weak-line groups in the earlier types so the same name was applied. Note that the differences are similar to those in the high-velocity stars although the magnitude of the differences is much smaller. About $15 \%$ of the late giants exhibit peculiarities in the $C N$ band as well as strong $C H$ and $\lambda 4226$. Some display stronger absorption between $\lambda 4145$ and $\lambda 4215$ with a different appearance in this region. The latter had been recognized on objective prism plates by Nassau and his associates who called them $\lambda 4150$ stars. The entire $C N$ band appears too strong for the strength of the hydrogen lines and of $\lambda 4077$. For the remaining stars the $C N$ band is too weak for the strength of $\lambda 4077$ and the trigonometric parallaxes. The $\lambda 4150$ stars have a larger dispersion in space velocities than the weak-line stars; the stars with weak $C N$ share the velocity characteristics of the high-velocity stars, although some have velocities as low as $40 \mathrm{~km} / \mathrm{sec}$.

After the publication of Roman's papers, Vyssotsky again classified the stars on his objective prism plates, dividing 810 of the spectra into two groups according to the strength of the G-band and $H \gamma$. His results for both the derived absolute marnitudes and the space motions were in remarkable agreement with Roman's results for the bright stars, showing that the relative number of stars in the two groups does not change with magnitude.

At the Vatican Conference Chalonge (1957) reported on Divan's work, which was still in progress. The few stars that had been measured showed a clear difference in the Chalonge parameters between Roman's weak- and 
strong-lined stars with the former differing from the mean in the direction of the subdwarfs. Later comparisons of the ultraviolet excesses as a function of velocity (Roman, 1963) and $[M / H]$ as a function of $U-B$ excess (Wallerstein and Carlson, 1960) showed a smooth change without discrete groupings.

Although astronomers no longer recognize a clean dichotomy in stellar populations, almost all of the bright stars are clearly Population I stars. Nevertheless, they do not form a homogeneous group. They differ in metal content and probably in age with the older stars having higher velocities and velocity dispersions.

\section{The Population of the High-Velocity Stars}

Because the high-velocity stars lag behind circular rotation and, hence, must move in elliptical orbits, thus crossing the interarm region, they were associated with Baade's Population II. The fact that globular clusters and RR Lyrae stars also have high velocities relative to the Sun strengthened this association, but most high-velocity stars belong to neither the halo nor the nuclear population. Among the stars discussed in this paper, the exceptions are the F-type subdwarfs and a group of A-type stars that appear normal on MK dispersion spectra.

Schwarzschild (1952) plotted the high-velocity stars from Miczaika's catalog (1940) on a Bottlinger diagram. Of the 555 stars plotted only 35 penetrate within 0.3 of the Sun's distance from the galactic center. On the other hand, they are not associated with the spiral arms, readily penetrating the interarm regions. As mentioned above, the spectra of the bright globular cluster giants do not resemble those of any high-velocity star (Baum, 1952; Roman, 1965). Keenan and Keller (1953) plotted an HR diagram for the high-velocity stars based on spectroscopic parallaxes. They state: "When comparison is made with the steeply slanted giant branches for the stars in globular clusters, 'the distribution of the high-velocity stars could be described as intermediate between those of the cluster stars and those of the low-velocity stars." Gratton (1954) also attributed them to an intermediate group on the basis of their chemical compositions. In fact, the distribution is not dissimilar to that of the low-velocity stars and of old open clusters except for the scarcity of stars earlier than F (Roman, 1965).

The high-velocity stars are not a homogeneous group. To investigate the characteristics as a function of spectral type, the author analyzed the data in her high-velocity star catalog (Table 1). To avoid distortion of the mean eccentricities by poorly determined eccentricities near 1.0, stars with e $>0.98$ were omitted from those means but not from any other means; similarly, semi-major axes larger than $30 \mathrm{kpc}$ were omitted in the determination of the mean a. Peculiar A stars and metallic line stars were 
also omitted. They are few in number but do not differ significantly from the F-K1 stars. The orbits are computed on the assumptions that the velocity of the local standard of rest is $216 \mathrm{~km} / \mathrm{sec}$, the Sun is $8.2 \mathrm{kpc}$ from the center, and the orbits are Keplerian. A more realistic gravitational potential would increase the perigalactic distances but not change the relative values.

TABLE 1. Orbital characteristics of high-velocity stars

\begin{tabular}{lrrrrrrr}
\hline Class & $(1)$ & $(2)$ & $(3)$ & $(4)$ & $(5)$ & $(6)$ & $(7)$ \\
\hline B7-A4 & 12 & 98 & 166 & 0.5 & 8.5 & 3.8 & 17 \\
F -K1 & 408 & 36 & 121 & 0.5 & 8.6 & 3.9 & 4 \\
K2-K4 & 114 & 29 & 100 & 0.4 & 8.4 & 4.4 & 1 \\
K5-M4 & 35 & 23 & 76 & 0.3 & 7.6 & 4.7 & 1 \\
Subdwarfs & 20 & 54 & 207 & 0.8 & 6.3 & 0.9 & 30 \\
\hline
\end{tabular}

(1) Number of stars included

(2) Mean velocity perpendicular to the galactic plane without regard to sign

(3) Mean space velocity

(4) Mean eccentricity

(5) Mean semi-major axis

(6) Average perigalactic distance

(7) Percentage of stars omitted from averages

For the most part, the galactic orbits do not differ greatly, although the latest stars, both giants and dwarfs, have somewhat smaller average velocities and less eccentric orbits. As is well known, the subdwarf orbits mimic those of the RR Lyrae stars. The early A-type stars form a small but interesting group. Their velocities perpendicular to the galactic plane are larger than those for any other group but they are not from the nuclear region. Spectroscopically on low dispersion, these stars look like normal A-type dwarfs, and their tangential velocities were computed on that assumption. They also have extremely large radial velocities. They do not resemble RR Lyrae stars spectroscopically, and their orbits are not particularly eccentric.

By the time of the Vatican Conference, it was clear that the abundance of metals in stars correlated statistically with their space velocities. Metals are under-abundant in most high-veloctiy stars, but most are neither halo nor nuclear stars.

\section{References}

Adams, W. and Kohlshütter, A., 1914, Ap. J. 39, 341

Baade, W., 1944, Ap. J. 100, 137

Baum, W., 1952, Ap. J. 57, 222 
Berger, J., Canavagia, R., Chalonge, D., and Fringant, A., 1951, Compt. Rend. Acad. Scie. Paris, 232, 2079

Chalonge, D., 1957, Stellar Populations, (O'Connell, ed.), p.345, Vatican Observatory, North Holland Publ. Co.

Chamberlain, J. and Aller, L., 1951, Ap. J. 114, 52

Gratton, L., 1953, Colloqu. Int. d'Astrophys. à Liege, 419

Iwanoska, 1950, Bull. Astr. Obs. Torun, no. 9, 25

Keenan, P. and Keller, G., 1953, Ap. J. 113, 700

Keenan, P., Morgan, W., and Münch, G., 1948, A. J. 53, 194

Lindblad, B., 1922, Ap. J. 55, 85

Miczaika, G., 1940, Astron. Nachtr. 270, 249

Miczaika, G., 1950, Zeitschr. Astroph. 27, 1

Morgan, W., Keenan, P., and Kellman, E., 1943, An Atlas of Stellar Spectra, U. Chicago Press, Chicago

Oort, J., 1926, Groningen Publ. \#40

Popper, D., 1947, Ap. J. 105, 204

Reiz, A., 1954, Ap. J. 120, 342

Roman, N., 1950, Ap. J. 112, 554

Roman, N., 1954, A. J. 59, 307

Roman, N., 1955, Ap. J. Suppl. 2, 195

Roman, N., 1965, Stars and Stellar Systems, Vol. 5: Galactic Structure, p. 345, U. Chicago Press, Chicago

Schwarzschild, M. and Schwarzschild, B., 1950, Ap. J. 112, 248

Schwarzschild, M., 1952, A. J. 57, 57

Schwarzschild, M., Searle, L., and Howard, R., 1955, Ap. J. . 122, 353

Schwarzschild, M., Sptizer, L., and Wildt, R., 1951, Ap. J. . 114, 398

Vyssotsky, A., 1950, Trans. IAU, 7, 448

Vyssotsky, A., and Skumanich, A., 1953, A. J. 58, 96

Wallerstein, G. and Carlson, M., 1960, Ap. J., 132, 276

PAGEL: It is somewhat ironical to not note that HD140283, one of the two classical "subdwarfs", has had its parallax revised downward from about 30 to 12 mas, making it a subgiant as was previously indicated by model atmosphere analyses giving the surface gravity. 